\title{
Oral health promotion reduces plaque and gingival bleeding in the short term
}

\author{
Does oral health promotion improve oral hygiene and gingival health?
}

\begin{abstract}
Watt RG, Marinho VC. Does oral health promotion improve oral hygiene and gingival health? Periodontol 2000 2005; 37:35-47

Data sources Cochrane Database of Systematic Reviews, Database of Abstracts of Reviews of Effects, Health Technology Assessment Database, UK National Health Service Economic Evaluation Database, Cochrane Central Register of Controlled Trials and Medline. There were no date limits or language restriction.

Study selection Systematic reviews and controlled trials (randomised or quasi-randomised) assessing reductions in dental plaque levels and/or gingival bleeding (gingivitis) and comparing health education/ health promotion interventions that did not involve clinical professional input or the use of pharmacological interventions, such as antiplaque agents were included. Studies involving only special groups, such as orthodontic or medically compromised patients or assessing only denture plaque, were excluded.
\end{abstract}

Data extraction and synthesis A range of data were extracted from systematic reviews and trials, quality assessment was undertaken, and a qualitative overview of the findings was provided.

Results Twenty-six potentially relevant studies were identified. Six reports of five systematic reviews and 13 trials were discussed: four studies were not assessed as one was published in Polish and three could not be located.

A wide range of educational and behavioural interventions were considered. These did not generally include clinical interventions and social or environmental approaches. The reviews have a number of limitations so their conclusions should be viewed with a degree of caution. However, the majority of studies achieved short-term reductions in plaque and gingival bleeding. Precise estimates of the improvement are difficult to assess because of the range and diversity of outcome measures used. The results of two meta-analyses indicate a reduction in plaque levels of $32-37 \%$.

Of 13 recently published trials evaluating educational interventions, five were set in schools, four focused on adults either in a clinical or workplace setting, three targeted older people, and one, infants. The design quality of the trials was variable. Allocation concealment was clearly described in two trials only, but blind outcome assessment was described in most of the trials and so were dropout rates. Other problems included a lack of controls, use of single blinding and relatively short follow-up.

Positive effects on plaque and/or bleeding outcomes were seen in eight studies with no difference in five studies, of which only two employed a control group. Nevertheless, for the two trials that compared various approaches, reductions in plaque and gingival bleeding were generally observed in all groups over the trial period. None of the studies produced a negative effect. Although all the studies evaluated educational interventions, there was no clear indication that any particular type or style of educational approach was more effective than any other.

Conclusions Reductions in plaque and gingival bleeding were seen in the short term in the majority of studies reviewed. The clinical and public health significance of these changes is, however, questionable. Future studies should use longer follow-up periods to assess whether

Address for correspondence: RG Watt, Department of Epidemiology and Public Health, University College London Medical School, 1-19 Torrington Place, London, UK. short-term beneficial changes are sustained. Other forms of oral health promotion require better quality evaluation if they are to be used to improve periodontal health.

\section{Commentary}

This thorough review confirms the notion that it is possible to achieve short-term reductions in plaque and gingival bleeding by means of health education interventions. The importance of these reductions is questionable, however, from the point of view of both individual and public health. It is also unclear how well the beneficial changes are sustained in the long run: studies with long follow-up periods are needed to find out the long-term effects.

This leads to the question of the role of health education among the various means for controlling plaque and gingival bleeding. Why should we educate our patients if the beneficial effects are likely to be short-lived only, and possibly unimportant?

First, the fact that there is no convincing evidence about the significance of health education does not exclude the possibility that beneficial effects exist. Studies on other health problems have revealed that a favourable change in health behaviour can follow a lengthy process that includes several stages. ${ }^{1,2}$ It is possible that a health education intervention contributes to the process even in the case that no change in behaviour can be seen during a short period of time.

Second, as experts, we have an ethical imperative to tell patients about how they might control their health better. In Finland, the obligation of informing patients has even been included in the law on the status and rights of the patient. An individual who has plaque and gingival bleeding is entitled to be informed about the problem and the significance of oral hygiene in a way that is understandable and clear.

The term "oral health promotion" has been used in the title of the review. All the reviews and individual studies that were assessed evaluated health education interventions. Consequently, it might have been preferable to use the term "health education". According to the contemporary concept of health promotion, ${ }^{3}$ developing personal skills, although an important aim of health education, is only one out of five areas of health promotion action. The others are building a healthy public policy, creating supportive environments, strengthening community actions, and reorienting health services. The effects of the latter approaches are not easy to evaluate, which may explain why no such appraisal was available for this review.

\section{Hannu Hausen \\ Institute of Dentistry, University of Oulu, Oulu, Finland}

1. Vallis M, Ruggiero L, Greene G, Jones H, Zinman B, Rossi S, et al. Stages of change for healthy eating in diabetes: relation to demographic, eating-related, health care utilization, and psychosocial factors. Diabetes Care 2003; 26:1468-1474.

2. Prochaska JO, Velicer WF, Prochaska JM, Johnson JL. Size, consistency, and stability of stage effects for smoking cessation. Addict Behav 2004; 29:207-213.

3. Anonymous. Ottawa charter for health promotion. Can J Publ Health 1986; 77:425-430.

Evidence-Based Dentistry (2005) 6, 31.

doi:10.1038/sj.ebd.6400325 